\title{
Einstein's Pseudo-Tensor in $\boldsymbol{n}$ Spatial Dimensions for Static Systems with Spherical Symmetry
}

\author{
Frank R. Tangherlini \\ P. O. Box 928211, San Diego, CA 92192, USA \\ Email: frtan96@gmail.com
}

Received May 25, 2013; revised June 27, 2013; accepted July 21, 2013

Copyright (C) 2013 Frank R. Tangherlini. This is an open access article distributed under the Creative Commons Attribution License, which permits unrestricted use, distribution, and reproduction in any medium, provided the original work is properly cited.

\begin{abstract}
It was noted earlier that the general relativity field equations for static systems with spherical symmetry can be put into a linear form when the source energy density equals radial stress. These linear equations lead to a delta function energymomentum tensor for a point mass source for the Schwarzschild field that has vanishing self-stress, and whose integral therefore transforms properly under a Lorentz transformation, as though the particle is in the flat space-time of special relativity (SR). These findings were later extended to $n$ spatial dimensions. Consistent with this SR-like result for the source tensor, Nordström and independently, Schrödinger, found for three spatial dimensions that the Einstein gravitational energy-momentum pseudo-tensor vanished in proper quasi-rectangular coordinates. The present work shows that this vanishing holds for the pseudo-tensor when extended to $n$ spatial dimensions. Two additional conse- quences of this work are: 1) the dependency of the Einstein gravitational coupling constant $\kappa$ on spatial dimensionality employed earlier is further justified; 2) the Tolman expression for the mass of a static, isolated system is generalized to take into account the dimensionality of space for $n \geq 3$.
\end{abstract}

Keywords: Field Equations; Point Particle; Dimensionality of Space; Einstein's Pseudo-Tensor

\section{Introduction}

Some years ago, the author [1] obtained Einstein's field equations for static systems with spherical symmetry in $n$ spatial dimensions, and one time dimension, which led to a generalization of Schwarzschild's solution for three spatial dimensions. An earlier investigation by the author [2] had shown that for this symmetry with $n=3$, and for a suitable choice of coordinates, when the field equations were suitably rewritten, they were linear. Other cases for which the field equations are linear are mentioned, but these fall outside the scope of this work. The linearity which will be dealt with here is associated with the fact that for the source tensor, energy density equals radial stress, i.e., in the notation to be employed below, $T_{0}^{0}=T_{1}^{1}$, a condition that is obviously satisfied for the homogenous solution. This linearity explains why the solution with an electrically charged source, the Reissner-Nordström solution, for which the Maxwell energymomentum tensor satisfies this condition, as well as the solution that includes the cosmological term, superimposes on the Schwarzschild solution for a point mass, so that for the standard line element $(c=1)$, $d s^{2}=g_{00} d t^{2}+g_{11} d r^{2}-r^{2} d \theta^{2}-r^{2} \sin ^{2} \theta d \phi^{2}$, one has $g_{00}=1-2 G m / r+G e^{2} / r^{2}-\Lambda r^{2} / 3$, with $g_{11}=-\left(g_{00}\right)^{-1}$. This remarkable linearity enabled the author to extrapolate to the origin to find the source tensor for a point mass in terms of the Dirac delta function. Thus, for the source tensor $T_{v}^{\mu}$ of a point particle source, one has

$$
\begin{aligned}
& T_{v}^{\mu}=\operatorname{diag}(1,1,-1 / 2,-1 / 2) m \delta(r) / 2 \pi r^{2}, \\
& (\mu, v=0,1,2,3),
\end{aligned}
$$

where $\delta(r)$ is the radial delta function. This tensor has the important property that the trace of the spatial stresses vanishes, $T_{i}^{i}=0$. (Latin indices run 1, 2, 3, and Einstein's summation convention holds.) Because of this vanishing of the self-stress, see, e.g., Panofsky and Phillips [3], also Rohrlich [4], if one rewrites $T_{v}^{\mu}$ in terms of quasi-rectangular coordinates (this will be done in sect. 3 for $n$ dimensions), then upon making a Lorentz transformation for a "boost" with velocity $\boldsymbol{v}$, and integrating for the energy and momentum of the point particle, one finds $E=m \gamma, \boldsymbol{p}=m \gamma \boldsymbol{v}$, unlike the case for the classical electron based solely on the Maxwell energy-momentum tensor for which, as is well-known, the self-stress does not vanish, and one does not obtain the correct special relativistic values for the energy and momentum in the 
absence of compensating stresses.

The surprising consistency with special relativity (SR) of the delta function energy-momentum tensor for a point mass source for the Schwarzschild field raises the interesting question about the gravitational energy that might be associated with the solution. If one thinks of SR as the limiting case when the Einstein coupling constant $\kappa$ vanishes, where $\kappa=8 \pi G$ for three spatial dimensions, then it would seem reasonable that in this quasi-rectangular coordinate system, the Einstein gravitational energy-momentum pseudo-tensor should vanish for the Schwarzschild solution, if the analogy with SR continues to hold. This was indeed found to be the case by Nordström [5], and by Schrödinger [6], albeit without reference to SR. Nordström showed that the pseudo-tensor vanished for $g_{00} g_{11}=$ constant, and explicitly showed that the pseudo-tensor energy density vanished for this case, while Schrödinger, in an independent analysis, found that all the components of the pseudo-tensor vanished. In an investigation of the Reissner-Nordström solution for a charged particle in [7], the author calculated the pseudotensor in the same quasi-rectangular coordinates for the more general case in which $g_{00} g_{11} \neq$ constant, and found

$$
2 \kappa(-g)^{1 / 2} t_{v}^{\mu}=2 r^{-1}\left(-g_{00} g_{11}\right)_{, r}^{1 / 2}\left(1+g_{11}\right) \Phi_{j}^{i},
$$

where $\Phi_{0}^{0}=1, \Phi_{0}^{i}=\Phi_{i}^{0}=0, \Phi_{j}^{i}=-\frac{1}{2} n_{i} n_{j}+\delta_{i j}$, where $n_{i}=x^{i} / r$. Thus it is clear that the constancy of $g_{00} g_{11}$ is sufficient for the pseudo-tensor to vanish in this quasirectangular coordinate system. It should be emphasized, however, that if one transforms to the quasi-rectangular coordinates associated with the isotropic form of the above line element, for which the spatial metric is conformally flat, the pseudo-tensor does not vanish, and in particular, outside the Schwarzschild event horizon, the energy density falls off as the fourth power of the radial coordinate, just as does the Maxwell tensor for a charged particle. However, a further discussion of this wellknown problem concerning the pseudo-tensor, is outside the scope of this work, and will be taken up in a later work.

Returning then to the situation at hand, the question naturally arises as to whether or not this surprising SR behavior of the point mass tensor, associated with the vanishing of the self-stress and the vanishing of the Einstein pseudo-tensor, continues to hold after one extends the Schwarzschild solution to $n$ spatial dimensions? Thus, in Section 2, after recapitulation of the field equations for static systems with spherical symmetry for arbitrary $n$ as given earlier [1], in which there will be a clarification of the form given there for Einstein's gravitational coupling constant, i.e., its dependency on the dimensionality of space, and also there will be given a correction to one of the terms in the field equations that contained a typographical sign error, the $n$ dimensional generalization of (1) will be given and discussed. In Section 3, the Einstein pseudo-tensor for $n$ dimensions will be given, and the results will be shown to be consistent with the findings for three dimensions. In Section 4, there are concluding remarks.

\section{The Field Equations in $\boldsymbol{n}$ Spatial Dimensions}

The field equations were given in [1], but are given here again for convenience, and because one of the field equations (here individually numbered) contained a sign error of typographical origin, and is given here in corrected form. The line element, with a change in notation for the angles, is given by

$$
\begin{aligned}
d s^{2}= & \mathrm{e}^{v} d t^{2}-\mathrm{e}^{\lambda} d r^{2} \\
& -r^{2}\left(d \theta_{2}^{2}+\sin ^{2} \theta_{2} d \theta_{3}^{2}+\cdots+\prod_{\ell=2}^{\ell=n-1} \sin ^{2} \theta_{\ell} d \theta_{n}^{2}\right),
\end{aligned}
$$

where $g_{00}(r)=\mathrm{e}^{v}$ and $g_{11}(r)=-\mathrm{e}^{\lambda}$. After a standard, albeit lengthy, calculation for the field equations in mixed form, $G_{v}^{\mu}=-\kappa_{n} T_{v}^{\mu}$, where the form for $\kappa_{n}$ is irrelevant at this point, and will be discussed later below, the equations reduce to

$$
\begin{aligned}
& (n-1) \frac{\exp [-\lambda]}{2}\left[-\frac{\lambda^{\prime}}{r}+\frac{n-2}{r^{2}}\right]-\frac{(n-1)(n-2)}{2 r^{2}}=-\kappa_{n} T_{0}^{0}, \\
& (n-1) \frac{\exp [-\lambda]}{2}\left[\frac{v^{\prime}}{r}+\frac{(n-2)}{r^{2}}\right]-\frac{(n-1)(n-2)}{2 r^{2}}=-\kappa_{n} T_{1}^{1}, \\
& \frac{\exp [-\lambda]}{2} \\
& {\left[v^{\prime \prime}+\frac{v^{\prime 2}}{2}-\frac{\lambda^{\prime} v^{\prime}}{2}+\frac{(n-3)(n-2)}{r^{2}}-\frac{(n-2)\left(\lambda^{\prime}-v^{\prime}\right)}{r}\right]} \\
& -\frac{(n-2)(n-3)}{2 r^{2}}=-\kappa_{n} T_{2}^{2},
\end{aligned}
$$

and $T_{2}^{2}=T_{3}^{3}=\cdots=T_{n}^{n}$ because of spherical symmetry. In [1], in the third equation of the group labeled (3.3), there was a plus sign before the fifth term in the square parentheses instead of a minus sign, given now correctly above as $-(n-2)\left(\lambda^{\prime}-v^{\prime}\right) / r$. Upon setting $T_{0}^{0}=T_{1}^{1}$, as is trivially the case for the vacuum, it follows that $\lambda^{\prime}+v^{\prime}=0$, from which, for suitable normalization assuming asymptotic flatness, one has $g_{00} g_{11}=-1$. Then, upon setting $g_{00}=1+2 U$, Equations (4)-(6) reduce to the following two linear equations

$$
\frac{(n-1)}{r^{n-1}} \frac{\mathrm{d}}{\mathrm{d} r}\left(r^{n-2} U\right)=-\kappa_{n} T_{0}^{0}=-\kappa_{n} T_{1}^{1},
$$




$$
\nabla_{n}^{2} U+\frac{n-3}{r^{n-1}} \frac{\mathrm{d}}{\mathrm{d} r}\left(r^{n-2} U\right)=-\kappa_{n} T_{2}^{2},
$$

Where $\nabla_{n}^{2}$ is the radial LaPlacian for $n$ dimensions, $\mathrm{d}^{2} U / \mathrm{d} r^{2}+((n-1) / r) \mathrm{d} U / \mathrm{d} r$. The stress equilibriumequation that follows from the contracted Bianchi identities, and physically from the covariant energy-momentum conservation law, for the static case under consideration (i.e., with $T_{0}^{0}=T_{1}^{1}$ ) is given by

$$
\frac{1}{r^{n-1}} \frac{\mathrm{d}}{\mathrm{d} r}\left(r^{n-1} T_{1}^{1}\right)-\frac{n-1}{r} T_{2}^{2}=0 .
$$

In [1] it was assumed that

$$
\kappa_{n}=(n-1) \omega_{n} G \text {, }
$$

where $\omega_{n}=2 \pi^{n / 2} / \Gamma(n / 2)$ is the total solid angle, or area of the unit sphere, in $n$ spatial dimensions. However, the factor $(n-1))$ was introduced solely on the basis that the left hand side of the field equations for $G_{0}^{0}$ and $G_{1}^{1}$ vanished for $n=1$, and it seemed reasonable that the right hand side, proportional to $T_{0}^{0}$ and $T_{1}^{1}$ respectively, should vanish as well. Further, it was noted that combining the factor $(n-1)$ for $n=3$ with $\omega_{3}=4 \pi$ gives the correct Einstein numerical factor of $8 \pi$. More recently, it was recognized that instead of this somewhat ad hoc way of obtaining the factor $(n-1)$, there is an argument based on the signature difference for the metric tensor. Thus, choose coordinates such that the metric tensor is locally diagonalized, and the individual terms normalized to plus or minus unity. Let $N_{+}$be the number of positive metric coefficients, and $N_{-}$the number of negative metric coefficients, the signature difference is $\left(N_{+}-N_{-}\right)$. (Note, this is sometimes referred to as the signature itself.) For a time-like metric $\left(N_{+}-N_{-}\right)$yields $(1-n)=-(n-1)$, and clearly for a space-like metric, $\left(N_{+}-N_{-}\right)$yields $(n-1)$, and hence if the Einstein the field equations are written as

$$
G_{v}^{\mu} \equiv R_{v}^{\mu}-\frac{1}{2} \delta_{v}^{\mu} R=\left(N_{+}-N_{-}\right) \omega_{n} G T_{v}^{\mu},
$$

they hold in this form, not only for $n$ spatial dimensions, but independently of whether or not the metric is spacelike, or time-like; the minus sign that appears, or doesn't appear, on the right hand side is automatically taken care of by this signature-dependent form of the coupling constant. Thus (11) suggests that instead of the definition of $\kappa_{n}$ in (10), it is preferable to define it as

$\kappa_{n}=\left(N_{+}-N_{-}\right) \omega_{n} G$, but it will not be used here. Further discussion is in the concluding section.

It is readily shown that the solution to the homogeneous Equations (7) and (8) for $n \geq 2$ is

$U=-2 G m / r^{n-2}$, as given in [1], so that

$g_{00}=-g^{11}=\left(1-2 G m / r^{n-2}\right)$. To obtain the delta function

expression for the $T_{v}^{\mu}$ of a point particle, it is convenient to introduce the step function $\varepsilon(r)=1, r>0, \varepsilon(0)=0$, and set $U=-G m \varepsilon(r) / r^{n-2}$. Then from Equation (7) one has

$$
m \varepsilon^{\prime}(r) / r^{n-1}=\omega_{n} T_{0}^{0},
$$

and since $\varepsilon^{\prime}(r)=2 \delta(r)$, one has

$T_{0}^{0}=2 m \delta(r) / \omega_{n} r^{n-1}=T_{1}^{1}$. To obtain, $T_{2}^{2}=\cdots=T_{n}^{n}$, it is convenient to use (9) and $\delta^{\prime}(r)=-\delta(r) / r$ to obtain $T_{2}^{2}=-2 m \delta(r) /(n-1) \omega_{n} r^{n-1}$. Hence the energy-momentum tensor for the point mass in $n$ dimensions, $n \geq 2$, is given by

$$
T_{v}^{\mu}=\operatorname{diag}\left(1,1,-\frac{1}{n-1}, \cdots,-\frac{1}{n-1}\right) \frac{2 m \delta(r)}{\omega_{n} r^{n-1}},
$$

as obtained in [1], albeit with $\varepsilon^{\prime}(r)$ used there replaced with $2 \delta(r)$ here. As was true for the $n=3$ case, the trace of the spatial stresses vanishes,

$T_{i}^{i}=T_{1}^{1}+(n-1) T_{2}^{2}=0$. Upon transforming to quasi-rectangular coordinates, $r=\left(\delta_{i j} \bar{x}^{i} \bar{x}^{j}\right)^{1 / 2}$, the energy-momentum tensor takes form

$$
\bar{T}_{0}^{0}=T_{0}^{0}, \bar{T}_{j}^{i}=T_{2}^{2} \delta_{i j}+\left(T_{1}^{1}-T_{2}^{2}\right) \mathrm{X}_{i} \mathrm{X}_{j},
$$

here $\mathrm{X}_{i}=\bar{x}^{i} / r$, and $T_{2}^{2}=\cdots=T_{n}^{n}$ has been used. Since $\mathrm{X}_{i} \mathrm{X}_{i}=1$, the trace of the spatial stresses in the quasi- rectangular coordinates is given by

$\bar{T}_{i}^{i}=T_{1}^{1}+(n-1) T_{2}^{2}=0$, as must be the case since the spatial trace is invariant under purely spatial transformations. Thus, not only in three spatial dimensions, but in $n$ spatial dimensions, the energy-momentum tensor of the point particle source for the generalized Schwarzschild field transforms properly under a pure Lorentz transformation to yield the correct SR energy-momentum relations for a particle of mass $m$. However, the question arises as to whether the Einstein pseudo-tensor continues to vanish for $g_{00} g_{11}=$ constant ? This will be shown to be the case in the next section.

\section{Einstein's Pseudo-Tensor in $\boldsymbol{n}$ Spatial Dimensions}

The line element is that in (3), and it is necessary to convert it to quasi-rectangular coordinates to obtain the appropriate metric tensor to use in calculating the pseudotensor. This is most simply accomplished by rewriting $g_{11} d r^{2}$ as $\left(1+g_{11}\right) d r^{2}-d r^{2}$, and then combining the $-d r^{2}$ term with the angular terms to give the contribution to the spatial metric as $-\delta_{i j} d x^{i} d x^{j}$, where the bars over the spatial coordinates have been dropped, so that at this point the spatial line element is

$\left(1+g_{11}\right) d r^{2}-\delta_{i j} d x^{i} d x^{j}$. Then, using $d r=\mathrm{X}_{i} d x^{i}$, so that $d r^{2}=\mathrm{X}_{i} \mathrm{X}_{j} d x^{i} d x^{j}$, and setting $g_{00}=\mathrm{e}^{\alpha}, g_{11}=-\mathrm{e}^{\lambda}$, the line element takes the form

$$
d s^{2}=\mathrm{e}^{\alpha} d t^{2}-\left(\delta_{i j}-\left(1-\mathrm{e}^{\lambda}\right) \mathrm{X}_{i} \mathrm{X}_{j}\right) d x^{i} d x^{j},
$$


and therefore the spatial metric is

$g_{i j}=-\delta_{i j}+\left(1-\mathrm{e}^{\lambda}\right) \mathrm{X}_{i} \mathrm{X}_{j}$. The contravariant spatial metric is readily found to be $g^{i j}=-\delta_{i j}+\left(1-\mathrm{e}^{-\lambda}\right) \mathrm{X}_{i} \mathrm{X}_{j}$, and likewise it is readily shown that in this new coordinate system $(-g)^{1 / 2}=\exp ((\alpha+\lambda) / 2)$. The Einstein pseudotensor is given by

$$
\begin{aligned}
& 2 \kappa_{n} \sqrt{-g} t_{v}^{\mu} \\
= & \Gamma_{\gamma \delta}^{\mu}\left(\sqrt{-g} g^{\gamma \delta}\right)_{, \nu}-(\ln \sqrt{-g})_{, \gamma}\left(\sqrt{-g} g^{\gamma \mu}\right)_{, \nu}+\delta_{v}^{\mu} \mathcal{L},
\end{aligned}
$$

where $\mathcal{L}$ is $2 \kappa_{n}$ times the Lagrangian density, and is given by

$$
\mathcal{L}=\sqrt{-g} g^{\gamma \delta}\left(\Gamma_{\gamma \beta}^{\eta} \Gamma_{\eta \delta}^{\beta}-\Gamma_{\gamma \delta}^{\eta} \Gamma_{\eta \beta}^{\beta}\right) .
$$

The Christoffel symbols of the second kind for the metric associated with (15) are

$$
\begin{gathered}
\Gamma_{00}^{0}=0, \Gamma_{i j}^{0}=0, \Gamma_{0 i}^{0}=\frac{1}{2} \alpha^{\prime} \mathrm{X}_{i}, \\
\Gamma_{j 0}^{i}=0, \Gamma_{00}^{i}=\frac{1}{2} \mathrm{e}^{(\alpha-\lambda)} \alpha^{\prime} \mathrm{X}_{i}, \Gamma_{j k}^{i} \\
=\mathrm{X}_{i}\left(\frac{1-\mathrm{e}^{-\lambda}}{r}\right)\left[\left(\delta_{j k}-\mathrm{X}_{j} \mathrm{X}_{k}\right)+\frac{1}{2} \lambda^{\prime} \mathrm{X}_{j} \mathrm{X}_{k}\right] .
\end{gathered}
$$

Also, from (18) and (19),

$\Gamma_{\gamma \beta}^{\beta}=\left\{\Gamma_{0 \beta}^{\beta}, \Gamma_{i \beta}^{\beta}\right\}, \Gamma_{0 \beta}^{\beta}=0, \Gamma_{i \beta}^{\beta}=\Gamma_{i 0}^{0}+\Gamma_{i j}^{j}=\frac{1}{2}\left(\alpha^{\prime}+\lambda^{\prime}\right) \mathrm{X}_{i}$. This also follows directly using $\Gamma_{\gamma \beta}^{\beta}=\partial_{\gamma} \ln (-g)^{1 / 2}$. Because the system is static, and time derivatives as well as terms in $g^{0 i}$ vanish, the gravitational energy density is proportional to $\mathcal{L}$. Hence upon carrying out the analysis, it follows that

$$
2 \kappa_{n} \sqrt{-g} t_{0}^{0}=\mathcal{L}=\mathrm{e}^{(\alpha+\lambda) / 2}\left(\frac{\alpha^{\prime}+\lambda^{\prime}}{2 r}\right)\left(1-\mathrm{e}^{-\lambda}\right)(n-1) .
$$

Note that the right hand side vanishes for $n=1$, consistent with the fact that both $\kappa_{1}$ and (4) and (5) vanish. Since $t_{i}^{0}=t_{0}^{i}=0$, the only remaining terms left are the stresses $t_{j}^{i}$ which from (16) can be written as a sum of two terms, $2 \kappa_{n}(-g)^{1 / 2} t_{j}^{i}=K_{j}^{i}+\delta_{j}^{i} \mathcal{L}$, and again, carrying out the analysis, it follows that

$$
K_{j}^{i}=-\mathrm{e}^{(\alpha+\lambda) / 2}\left(\frac{\alpha^{\prime}+\lambda^{\prime}}{2 r}\right)\left(1-\mathrm{e}^{-\lambda}\right)\left(\delta_{i j}+(n-2) \mathrm{X}_{i} \mathrm{X}_{j}\right),
$$

which upon combining with $\delta_{j}^{i} \mathcal{L}$ yields finally

$$
\begin{aligned}
& 2 \kappa_{n} \sqrt{-g} t_{j}^{i} \\
= & \mathrm{e}^{(\alpha+\lambda) / 2}\left(\frac{\alpha^{\prime}+\lambda^{\prime}}{2 r}\right)\left(1-\mathrm{e}^{-\lambda}\right)\left(\delta_{i j}-\mathrm{X}_{i} \mathrm{X}_{j}\right)(n-2) .
\end{aligned}
$$

It should be noted that the right hand side, although it does not explicitly exhibit the quantity $(n-1)$, nevertheless vanishes for $n=1$, since under these circumstances there is only $\delta_{11}=1$ and $\mathrm{X}_{1} \mathrm{X}_{1}=1$, and hence the term in parentheses involving the difference of these two quantities vanishes. Also if one takes the trace, $t_{i}^{i}$, then one has $\left(\delta_{i i}-\mathrm{X}_{i} \mathrm{X}_{i}\right)=(n-1)$, so in this case the factor appears explicitly. Moreover, since there is spherical symmetry, the diagonal terms $t_{\ell}^{\ell}$ (no sum) are $n^{-1}$ times the trace, and since there are no off-diagonal terms for $n=1$, the vanishing for $n=1$ follows. Since both $t_{0}^{0}$ and $t_{j}^{i}$ are proportional to $\left(\alpha^{\prime}+\lambda^{\prime}\right)=\left(\ln \left(-g_{00} g_{11}\right)\right)_{, r}$ the pseudo-tensor vanishes for $g_{00} g_{11}=$ constant, so that the findings of Nordström [5] and Schrödinger [6] continue to hold in $n$ dimensions. Thus the tensor for the point mass in $n$ dimensions not only transforms properly under a Lorentz transformation, but, in this coordinate system, it is unaccompanied by gravitational energy and momentum, as if it were in the flat space-time of SR; indeed, since one can obviously set $(-g)^{1 / 2}=1$, the spacetime volume element is simply $d t d^{n} x$ as in SR. Regrettably, the underlying physical reason for these surprising results is still unknown.

There is an interesting property of the Einstein pseudotensor for static systems that was found by Papapetrou [8] for the case $n=3$, and is known as Papapetrou's identity. He found that $t_{0}^{0}-t_{i}^{i}=0$. In a study of the source of the Schwarzschild field. the author [9] observed, using Euler's theorem, and the general form for the pseudo-tensor from the action principle, that the trace of the spatial stresses, for a static system, in the notation of this paper, satisfies

$$
t_{i}^{i}=(n-2) t_{0}^{0},
$$

and observed that therefore the Papapetrou identity only holds in three spatial dimensions. Examination of (20) and (22) show that they obey (23), and this agreement can be seen as a check of the calculations leading to these two equations. The generalization of the Papapetrou identity given in (23), together with other assumptions given below, leads to a generalization of the Tolman [10] equations for the mass of an isolated static system. Thus, for $n \geq 3$, one has

$$
\begin{aligned}
m & =\int \sqrt{-g}\left(T_{0}^{0}+t_{0}^{0}\right) \mathrm{d}^{n} x=\int \sqrt{-g}\left(T_{0}^{0}+(n-2)^{-1} t_{i}^{i}\right) \mathrm{d}^{n} x \\
& =\int \sqrt{-g}\left(T_{0}^{0}-T_{i}^{i}(n-2)^{-1}\right) \mathrm{d}^{n} x,
\end{aligned}
$$

where the last integral has made use of the fact that since $\left(\sqrt{-g}\left(T_{j}^{i}+t_{j}^{i}\right)\right)_{, i}=0$, upon multiplication by $x^{j}$, and the use of the divergence theorem, together with sufficient fall-off of the stresses so that the surface integral of $x^{j}\left(\sqrt{-g}\left(T_{j}^{i}+t_{j}^{i}\right)\right)$ vanishes, at infinity, one has that $\int \sqrt{-g}\left(T_{i}^{i}+t_{i}^{i}\right) \mathrm{d}^{n} x=0$.

\section{Concluding Remarks}

The primary purpose of this work was to investigate 
whether the Einstein pseudo-tensor continued to vanish in $n$ spatial dimensions as it had for 3 dimensions for $g_{00} g_{11}=$ constant. As the above work shows, this is indeed the case, so that the vanishing is invariant with respect to a change of spatial dimensionality. Since this condition on the metric tensor via the field equations is directly tied to the requirement on the energy-momentum tensor that $T_{0}^{0}=T_{1}^{1}$, there is obviously a physical reason underlying this influence on the metric tensor, as well as the resulting linearity of the field equations which continues to hold in $n$ dimensions. However, as emphasized above, there is no known physical principle that leads one to expect these results. Equally interesting is the fact that when one transforms to isotropic coordinates, one finds that the pseudo-tensor does not vanish for $T_{0}^{0}=T_{1}^{1}$, and, as is well-known, no longer does $g_{00} g_{11}=$ constant for the Schwarzschild solution. A further study is in preparation in which the pseudo-tensor for isotropic coordinates in $n$ spatial dimensions will be given, and compared with the results obtained in the coordinate system used here. Finally, it is of interest that the work strongly suggests that when one goes outside three spatial dimensions, one should allow for a dimensionality dependence of Einstein's coupling constant, since the standard value of the constant, $8 \pi G$, is obtained to insure agreement with Newtonian theory in the limit of weak fields for $n=3$. Interestingly, the proposed behavior of the coupling constant in this work impacts on string theory, since the latter, in later models, leads to a spatial dimensionality of $n=10$, and so naturally the question arises-how does this higher dimensionality influence the gravitational coupling constant for this theory? An answer to this question could facilitate a comparison of string theory with general relativity for higher dimensions, as well as three dimensions. Likewise to be compared are the energy-momentum tensors for gravitation in the two theories, bearing in mind that a spin-two theory would seem to predict a true tensor, whereas in accordance with the principle of general covariance, and the demand for a true conservation law, general relativity predicts a pseudo-tensor.

Also, it will be noted that unlike string theory, Einstein's field equations do not in themselves impose a restriction on the dimensionality of space, although to be sure, for $n=1$, according to (11), the gravitational coupling vanishes, while for $n=2$ outside the source, the homogeneous solution indicates space-time is flat, and one therefore does not have the Newtonian logarithmic potential; for $n \geq 3$, one has quasi-Newtonian behavior. The way $n=3$ arrived at in [1] which was by requiring that there be stable bound orbits in the generalized Schwarzschild field. This approach was a follow-up to the study of the dimensionality of space problem by Ehren- fest $[11,12]$ who studied the stability of orbits in Newtonian theory extended to $n$ dimensions. Ehrenfest's approach for $n \geq 3$ was later arrived at independently by Whitrow $[13,14]$, who justified the stability assumption by an appeal to the anthropic principle. Of interest to the present work is the fact that in [1], the pseudo-tensor is not involved, and so the question arises as to whether the behavior of the pseudo-tensor in $n$ dimensions has bearing on the dimensionality of space problem? A possible affirmative answer to this question seems to be provided by (23) that shows that Papapetrou's identity for static systems, $t_{0}^{0}=t_{i}^{i}$, only holds for $n=3$, and hence Tolman's expression for the mass of an isolated static system as well. Consequently, further studies of the behavior of Einstein's pseudo-tensor may lead to another way to tackle the dimensionality of space problem in the framework of general relativity, and perhaps give insight as to how to involve the stability principle in a less $a d$ hoc way.

\section{REFERENCES}

[1] F. R. Tangherlini, Nuovo Cimento, Vol. 27, 1963, pp. 636-651.

[2] F. R. Tangherlini, Physical Review Letters, Vol. 6, 1961, pp. $147-149$.

[3] W. H. Panofsky and M. Phillips, "Classical Electricity and Magnetism," 2nd Edition, Addison-Wesley, Reading, 1962.

[4] F. Rohrlich, "Classical Charged Particles," 3rd Edition, World Scientific, Singapore, 2007.

[5] G. Nordström, Proceedings of Amsterdam Academy of Sciences, Vol. 20, 1918, pp. 1238-1245.

[6] E. Schrödinger, Physikalische Zeitschrift, Vol. 19, 1918, pp. 4-7.

[7] F. R. Tangherlini, Nuovo Cimento, Vol. 26, 1962, pp. 497-524.

[8] A. Papapetrou, Proceedings of the Royal Irish Academy, Vol. A51, 1947, pp. 191-204.

[9] F. R. Tangherlini, Nuovo Cimento, Vol. 38, 1965, pp. 153-171.

[10] R. C. Tolman, "Relativity, Thermodynamics, and Cosmology," Clarendon Press, Oxford, 1934.

[11] P. Ehrenfest, Proceedings of the Amsterdam Academy of Sciences, Vol. 20, 1917, pp. 200-209.

[12] P. Ehrenfest, Annals of Physics (Leipzig), Vol. 61, 1920, pp. 440-446.

[13] G. J. Whitrow, British Journal for the Philosophy of Science, Vol. 6. 1955, pp. 13-31.

[14] G. J. Whitrow, "The Structure and Evolution of the Universe," Harper Torchbooks, New York, 1959, pp. 199201. 